\title{
Structural analysis of polymorphs of small organic molecules by 3D ED/MicroED

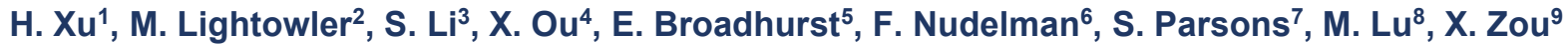 ${ }^{1}$ Department of Materials and Environmental Chemistry ${ }^{2}$ Stockholm University, ${ }^{3}$ Sun Yat-sen University, ${ }^{4}$ Sun Yat-sen University, ${ }^{5}$ The University of Edinburgh, ${ }^{6}$ The University of Edinburgh, ${ }^{7}$ The University of Edinburgh, ${ }^{8}$ Sun Yat-sen University, ${ }^{9}$ Stockholm University hongyi.xu@mmk.su.se
}

Polymorphism occurs when a material crystallizes into more than one distinct solid forms, which is commonly observed in organic chemistry. Polymorphs of small organic molecules differ in solubility, bioavailability and processing characteristics, affecting their properties and performances in applications such as opto-electronics, energy storage and pharmaceuticals[1].

Recent developments in 3D electron diffraction (3D ED) [2-5], also known as Micro-crystal electron diffraction (MicroED) [6,7], allowed rapid, atomic resolution structure determination of small organic molecules from nanoand micron-sized crystals. With our recent results, we show that 3D ED/MicroED is a powerful method for studying polymorphism of small organic molecules. The method can be used for 1) rapid structure determination of small organic molecules from small crystals $[5,8], 2)$ structural determination of complex and disordered crystal structures, 3) studying polymorph evolution of small organic molecules by in situ crystallization [9], and 4) investigating the growth mechanism of small organic molecules by capturing the earliest stages of the crystallization.

In order to further increase the throughput of polymorph analysis and phase identification, our group has developed serial electron diffraction (SerialED), where single diffraction patterns are collected from thousands of individual micro-crystals. By automated data collection enabled by software development, up to 3500 crystals can be studied per hour. SerialED, along with 3D ED/MicroED, will greatly accelerate polymorphic discovery and provide new possibilities to study reaction mixtures, dynamical processes and other applications where rapid structural analyses of materials are required.

1. Cruz-Cabeza, A. J. Reutzel-Edens, S. M. \& Bernstein, J. Facts and fictions about polymorphism. Chem. Soc. Rev. 44, 86198635 (2015).

2. Kolb, U. Gorelik, T. E. Mugnaioli, E. \& Stewart, A. Structural Characterization of Organics Using Manual and Automated Electron Diffraction. Polym. Rev. 50, 385-409 (2010).

3. Zhang, D. Oleynikov, P. Hovmöller, S. \& Zou, X. Collecting 3D electron diffraction data by the rotation method. Z. Für Krist. 225, (2010).

4. Wan, W. Sun, J. Su, J. Hovmöller, S. \& Zou, X. Three-dimensional rotation electron diffraction: software RED for automated data collection and data processing. J. Appl. Crystallogr. 46, 1863-1873 (2013).

5. Gemmi, M. et al. 3D Electron Diffraction: The Nanocrystallography Revolution. ACS Cent. Sci. 5, 1315-1329 (2019).

6. Shi, D. Nannenga, B. L. Iadanza, M. G. \& Gonen, T. Three-dimensional electron crystallography of protein microcrystals. eLife 2, e01345 (2013).

7. Nannenga, B. L. \& Gonen, T. The cryo-EM method microcrystal electron diffraction (MicroED). Nat. Methods 16, 369-379 (2019).

8. Jones, C. G. et al. The CryoEM Method MicroED as a Powerful Tool for Small Molecule Structure Determination. ACS Cent. Sci. 4, 1587-1592 (2018).

9. Broadhurst, E. T. et al. Polymorph evolution during crystal growth studied by 3D electron diffraction. IUCrJ 7, 5-9 (2020). 